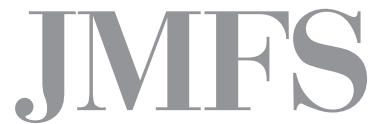

Journal of Management and Financial Sciences
Volume X

Issue 30 (December 2017)

pp. $115-136$

Warsaw School of Economics

Collegium of Management and Finance

Paweł Grunt

Collegium of Management and Finance

Warsaw School of Economics

\title{
Structured Analytic Techniques: Taxonomy and Technique Selection for Information and Intelligence Analysis Practitioners
}

\section{AbStRAct}

The purpose of this article is to propose a new taxonomy and selection of structured analytic techniques for information and intelligence analysis practitioners. The presented taxonomy and selection of structured analytic techniques are based on the author's experience in information and intelligence analysis as well as in training analysts in the use of those techniques. The presented classification and selection of techniques have resulted from the author's attempts to improve teaching and learning process of the analysts who were novice to structured analytic techniques. From the author's experience, a well-constructed taxonomy aids novice analysts in understanding a purpose of used techniques and selecting the best-suited technique for a given analytic problem. In the article the author will first offer definitions of analysis, analytic process and analytic spectrum. This will be followed by a review of the four general categories of analytic methods used in intelligence analysis. The author will further explain the origins, concepts and characteristics of both structured analysis and structured analytic techniques. Next, structured analytic techniques will be discussed in the context of the Intelligence Cycle. Following this, the author will review several existing taxonomies of structured analytic techniques. The article will conclude with a taxonomy and a selection of techniques for the information and intelligence analysis practitioners, based on a review of the literature augmented by the author's professional experience. 
Keywords: information, information analysis, analytic methods, analytic process, analytic spectrum, intelligence cycle, structured analysis, structured analytic techniques JEL Codes: Y800, C120, C530, D800, O210, O220, F530

\section{Introduction}

The technological and information revolution we have been witnessing over the last 20 years has caused an unprecedented amount of data and information to be available everywhere and for everyone. MindMetre Research in 2014 reported that one-nineteenth of all existing data had been produced after the year 2010. The same report noted that humanity sends 204 million electronic messages and exchanges $640000 \mathrm{~GB}$ of information every single minute ${ }^{1}$. One may say, we are drowning in a flood of information and data. The human brain has its working memory. It works like a computer memory, and because it has a limited capacity, it stores and processes a very limited amount of current information. According to George A. Miller, this short-term memory can store, on average, up to 7 plus or minus 2 bits of information. This number is called "the Miller's magic number" and is used to describe the human brain's cognitive capability for presented information ${ }^{2}$. For the intelligence and information analyst, information cognition is doubly complicated. First, there is a problem with volume. A human brain cannot possibly process the sheer amount of available information and data. Then, one cannot forget about data differentiation (SIGINT, GEOINT, biometric, video, etc.), which is expected to become wider every year as a result of the ongoing technological revolution.

The enormous amount and different types or forms of available data and information can cause "information overload". The information overload concept was introduced by Alvin Toffler, and describes a condition when the overabundance of the available information is paralysing the decision-making process instead of improving it ${ }^{3}$. In addition to the information overload, one has to consider the so-called "cognitive overload" of the brain. According to Lucy Jo Palladino, cognitive overload occurs

1 Forget information overload...the real challenge is content intelligence. Research Summary, MindMeetre, February 2014; http://www.mindmetreresearch.com/wp-content/uploads/2014/05/Paper_1_2014_ FINAL1.pdf, accessed 20.10.2016.

2 G.A. Miller, The Magical Number Seven, Plus or Minus Two, http://web.archive.org/web/2008072807 5223/www.musanim.com/miller1956/, accessed 24.02.2015.

3 A. Toffler, Future Shock, Random House, New York 1970, pp. 350-355. 
when we try to process large amounts of data while multitasking in our brain. The cognitive overload manifests itself with stress, indecisiveness, loss of concentration and a significant reduction in the brain's analytic capabilities ${ }^{4}$.

Some argue that the goal of intelligence activities is to gather as much data and information as possible to draw a picture of the situation as close as possible to the reality. States, military organizations and international corporations spend enormous amounts of money investing in top-end intelligence collection systems and platforms. However, in light of the aforementioned limited capabilities to process data already on hand, one should consider concentrating on the processing and analysis of data and information, rather than on collection. As B.E. Bensoussan and C.S. Fleisher claim, "In today's world of information overload, collecting more or new data or information is not (...) the key issue. Instead, examining and evaluating the information through analysis is the key to defining appropriate strategies and decisions. This process requires knowledge, skills, time, and effort"5.

One possible solution to the problem of limited capabilities of data and information processing is automated systems and software. Another is to significantly increase the number of employed analysts. As both solutions would be very expensive, improving existing procedures and analytic techniques is the solution to be considered, combined with introducing structured analytic techniques, and training analysts in using them.

The main task of contemporary supervisors and managers is to develop or participate in developing and defining tactics and strategies for organizations they represent. Being distinctive on the market is one of the basic conditions for an organization's success. In the context of strategy, it means developing unique and distinctive processes, relationships, and resources within organization, in order to ensure competitiveness on the market. Organizations that are able to develop these distinctive organizational competencies have the best chance to gain competitive advantage ${ }^{6}$. Organizations must have abilities to analyse and process information in order to effectively develop strategies. The analytic techniques facilitate the analysis of current and historical events in order to predict the future and thus, support the decision-making process resulting in organizational strategies. Structured analytic techniques are used within military organizations, intelligence, and law enforcement organizations. There is no reason against using them in business, local government and other sectors as well.

4 L.J. Palladino, Find Your Focus Zone. An Effective New Plan to Defeat Distraction and Overload, Atria Books, New York 2007.

5 B.E. Bensoussan, C.S. Fleisher, Analysis Without Paralysis. 12 Tools to Make Better Strategic Decisions, FT Press, Upper Saddle River, New Jersey 2013, p. 16.

6 Ibidem, p. 2. 
The purpose of this article is to provide a proposed taxonomy and selection of structured analytic techniques. The presented taxonomy and selection of structured analytic techniques is based on the author's experience in information and intelligence analysis as well as in training analysts in the use of those techniques. While training prospective analysts, the author noticed the importance of having a well-designed taxonomy. Such a taxonomy aids novice analysts in understanding the purpose of used techniques and selecting the best-suited technique for a given analytic problem. This significantly helps in the learning process and assists analysts when they start independently using those techniques in their work outside classrooms. In the author's judgement, the existing taxonomies of structured techniques should be complemented with two categories of techniques, i.e. environment scanning and assessment and source and information evaluation techniques. Those two categories are crucially important for an analyst in the early years of practising information and intelligence analysis. Thus, the proposed selection of techniques is tailored for the novice analysts and meant to assist them in selecting techniques. In order to achieve the stated purpose of the article, the author will start with offering definitions of analysis, analytic process and analytic spectrum. This will be followed by a review of the four general categories of analytic methods used in intelligence analysis. The author will further explain the origins, concepts and characteristics of both structured analysis and structured analytic techniques. Next, structured analytic techniques will be discussed in the context of the Intelligence Cycle. Following this, the author will review several existing taxonomies of the structured analytic techniques. The article will conclude with the taxonomy and a selection of techniques for the information and intelligence analysis practitioners, based on a review of the literature augmented by the author's professional experience.

\section{Analysis, Analytical Process and Analytic Spectrum}

Analysis can be defined as an activity of "the examination of a problem or phenomenon from different angles in order to understand or explain it" or as a product of the aforesaid activity in the form of an explanation or description ${ }^{7}$. NATO AJP-2 Allied Joint Doctrine for Intelligence, Counter-Intelligence and Security define analysis as a "step in the processing phase of the intelligence cycle in which information is subjected to review in order to identify significant facts for subsequent interpretation".

7 Słownik języka polskiego PWN. http://sjp.pwn.pl, accessed 21.10. 2016.

8 AJP-2, Allied Joint Doctrine for Intelligence, Counter-Intelligence and Security, Edition A version 1, 2014, p. 4-6. 
Analysis together with integration constitutes the third step in the processing stage of the Intelligence Cycle?.

Examining the above definitions, one can easily notice they do not mention or refer to the future, prediction or prognosis of events or an enemy's/competitor's actions. Those definitions only describe reactive analysis of past and current events; therefore, they do not describe all the stages and steps of the Intelligence Cycle.

The purpose of intelligence analysis is to evaluate, integrate and interpret information in order to identify threats and opportunities, and to provide a warning and reduce uncertainty for the decision maker ${ }^{10}$. However, its essence is predicting and forecasting. References to these activities are found in the definition of the scientific-research institute RAND, which defines intelligence analysis as a process by which the information collected about an enemy is used to answer tactical questions about current operations or to predict future behavior ${ }^{11}$. Defined in this way, the process of analysis already includes the full range (spectrum) analysis from "understanding or explanation" to "predicting" within the limits the analytical process is carried out (see Figure 1).

Figure 1. Analytic process

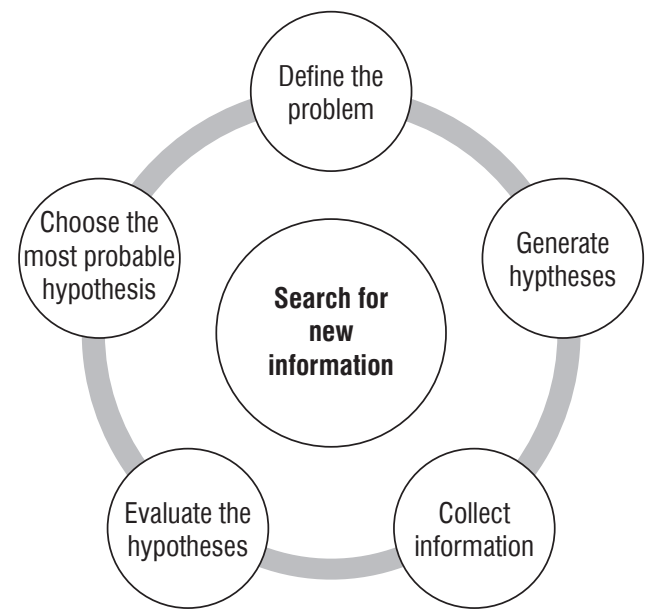

Source: R.J. Heuer, Psychology of Intelligence Analysis, Center for the Study of Intelligence, CIA 1999, p. 173.

9 Ibidem, pp. 4-7.

${ }^{10}$ B. Fischhoff, C. Chauvin, Intelligence Analysis: Behavioral and Social Scientific Foundations, The National Academies Press, Washington DC 2011, p. 2.

${ }^{11}$ Intelligence Analysis, RAND Corporation, http://www.rand.org/topics/intelligence-analysis.html, accessed 26.10. 2016. 
The analytic process consists of six steps: defining the problem, generating hypotheses, information gathering, evaluation of the hypotheses, selection of the most probable hypothesis, and continuous monitoring in search of new information ${ }^{12}$.

Define the problem. An analyst should start working on the problem with ensuring that (s)he is asking, and (s)he was asked, the right questions. He/she should not hesitate to turn to the authors or superiors to clarify doubts or provide additional detailed questions. Often, due to the fact that (s)he is an expert in the field, the analyst will see the problem differently and feel that the questions are inappropriate. In such cases, the analyst should not hesitate to give suggestions to the supervisor and the authors of the questions.

Generate hypotheses. In this step, all likely hypotheses are identified. Please note that at this stage we do not dismiss any of the hypotheses based on a lack of information or evidence to support it.

Gather information. While gathering information, the analyst cannot be limited only to information that is delivered to him/her. (S)he should actively seek facts and information using all available sensors and resources (Requests for InformationRFIs, or self-searching open sources). It should be noted that the analyst must seek information and facts to help evaluate all the hypotheses (both supporting them and questioning), and not only those that seem to him/her to be the most likely; and refrain from the evaluation of the hypotheses until the end of gathering information.

Evaluate the hypotheses. Based on the available information and assumptions the analyst develops arguments against each of the generated hypotheses.

Select the most probable hypothesis. Selection of the most probable hypothesis means refuting hypotheses (instead of confirming them). The hypothesis with the least amount of evidence against it is usually the most likely one to be true.

Continuously search for new information. While the analyst performs the steps of the analytic process, the situation concerning the analytical problem may change or the new information and facts either negating the most likely hypothesis, or supporting one of the rejected hypotheses may appear. For this reason, the analyst should seek new information and facts through the entire analytical process ${ }^{13}$.

In solving analytical problems and executing the analytic process, the analyst "moves" through the four stages of the analytic spectrum. In order to engage in the last, and at the same time, the most difficult stage of the analytic spectrum - estimative analysis - the analyst has to work through all the stages of the analytic spectrum. These successive stages: descriptive, explanatory, evaluative and estimative analysis,

12 R.J. Heuer, Psychology of Intelligence Analysis, Center for the Study of Intelligence, CIA 1999, p. 173.

13 R.J. Heuer, Psychology..., op.cit., pp. 173-177. 
guide the analyst in search for answers to the analytical problem (s)he is working on (see Figure 2).

\section{Figure 2. The Analytic Spectrum}

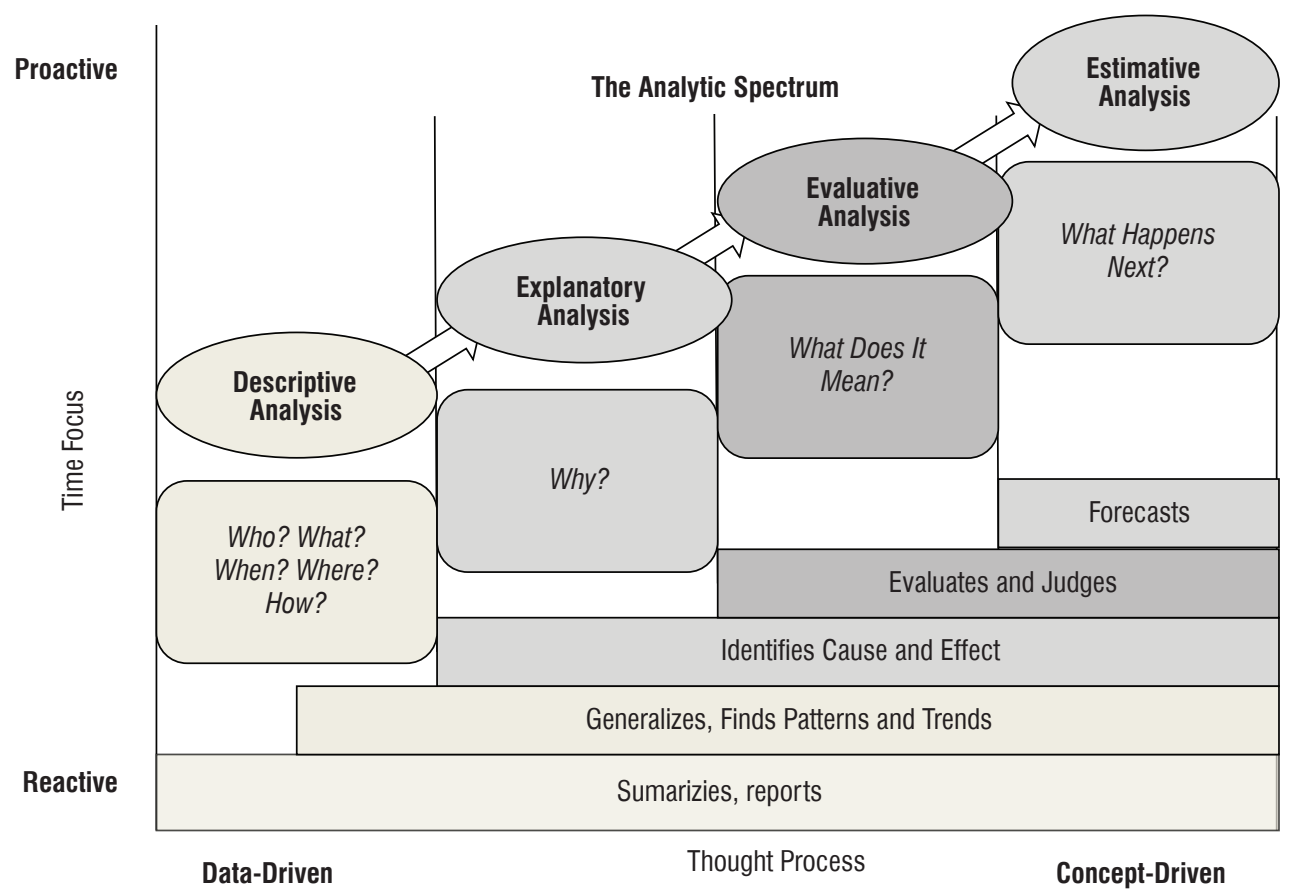

Source: K. Hibbs Pherson, R.H. Pherson, Critical Thinking for Strategic Intelligence, SAGE/CQPress, Los Angeles, 2013, pp. 46-48.

At the stage of descriptive analysis, the analyst answers the questions Who? What? How? When? and Where?, asked in relation to the analysed event, the situation, the problem or group of individuals. Products of the descriptive analysis inform and summarize the knowledge and information about people, objects, situations, etc. The descriptive analysis includes both Basic Intelligence and Current Intelligence products $^{14}$.

At the explanatory analysis stage, the analyst is looking for an answer to the question why the event, problem or situation has occurred. Target Intelligence and Current Intelligence products presenting trends are typical for this stage ${ }^{15}$.

${ }^{14}$ K. Hibbs Pherson, R.H. Pherson, Critical Thinking for Strategic Intelligence, SAGE/CQPress, Los Angeles, 2013, p. 48.

15 Ibidem. 
Descriptive and explanatory analysis are the necessary minimum two stages of each analytic process. But they are not sufficient to solve complex and complicated analytical problems demanding recommendations or forecasting, and therefore, the transition to the consecutive stages of the analytic spectrum.

The structured analytic techniques help the analyst to "make a transition" from one to the other stage of the analytic spectrum and facilitate analysis at those stages. In other words, after the stages of reactive analysis (descriptive and explanatory analysis, describing what has happened or is happening), the analyst is able to properly ${ }^{16}$ execute the proactive phase of the analytic spectrum answering the questions: What does it mean? (evaluative stage) and What could happen? (estimative stage) ${ }^{17}$.

Evaluative analysis uses logic, knowledge and analytical techniques to interpret and evaluate the data, examine the analytical problems presented by the recipient of the analytical product, and its products include Basic Intelligence, Current Intelligence and Target Intelligence products ${ }^{18}$. Estimative analysis is looking ahead into the future and, using different methods of analysis, tries to predict what will happen and what are the most probable future scenarios ${ }^{19}$.

\section{Analytic Methods}

Scientific methods have often been used for information and intelligence analysis in order to improve its processes and effectiveness. C.A. Mangio states "the belief that using the scientific method is the only way of knowing that consistently produces reliable knowledge motivates its application to analysis" 20 . The social and physical sciences methods are considered best suited for intelligence analysis ${ }^{21}$. Instead of using the scientific methods modified to suit the needs of intelligence analysts, Sherman Kent advocated a method specifically developed for intelligence ${ }^{22}$. The structured analysis is exactly such a method.

R.J. Heuer and R.H. Pherson organize analytical methods used in the field of intelligence analysis - scientific as well as developed specifically for the purpose of

16 'Properly' means in accordance with the principles and requirements of the chosen techniques, and avoiding cognitive biases and fallacies. This does not mean the accuracy of predictions and assessments of the situation - this cannot be guaranteed by any known analytic technique.

${ }^{17}$ K. Hibbs Pherson, R.H. Pherson, Critical ..., op.cit., pp. 48-49.

${ }_{18}$ Ibidem, p. 50.

19 Ibidem, p. 52.

${ }^{20}$ C.A. Mangio, Intelligence Analysis: Once Again, Air Force Research Laboratory Interim Report, February 2008, p. 13, http://www.dtic.mil/docs/citations/ADA520278, accessed 10.01.2017.

${ }^{21}$ Ibidem, p. 14

22 Ibidem. 
intelligence analysis, into four categories, and point out that the methods in these categories are different from each other, however, the boundaries between them are often blurred and hardly recognizable ${ }^{23}$ (see Figure 3).

Figure 3. Categories of methods used for intelligence analysis

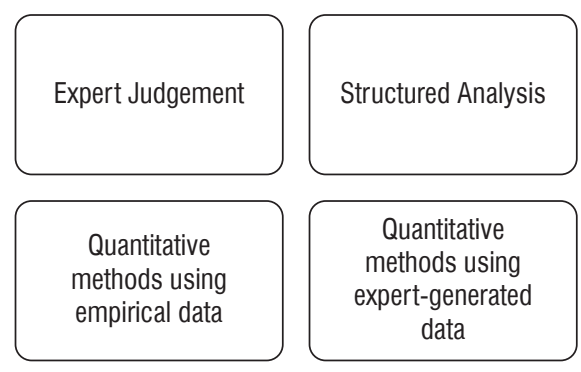

Source: the author's own study based on R.J. Heuer, R.H. Pherson, Structured Analytic Techniques for Intelligence Analysis, CQ Press, Washington DC 2011, p. 4.

Expert judgement, also called "traditional analysis", is the first category of analytical methods used in intelligence analysis. It is a collection of traditional analytic methods, which, among others, include methods such as evidentiary reasoning, case study and critical thinking. This category combines expert knowledge with critical thinking. The primary distinguishing feature of the expert judgement and structured analysis categories is that expert judgement relies mostly on the individual effort of the analyst, which remains in the head of the analyst until it is written in the form of a preliminary report. Education and training in the field of these methods is based on general education, and in particular in social sciences, combined with knowledge of the specifics like culture and language, of the analysed country or area ${ }^{24}$.

The second category consists of selected techniques, and is collectively referred to as structured analysis. Each technique belonging to this group is a gradual process, and its task is to present a thought process occurring in the head of the analyst in an understandable way for others, thus, allowing for understanding, debate, and criticism ${ }^{25}$. As a result, analysis becomes a team project, in which the transparency of the process analysis reveals other analysts its inconsistency or conflicting hypotheses. Structured

${ }^{23}$ R.J. Heuer, R.H. Pherson, Structured Analytic Techniques for Intelligence Analysis, CQ Press, Washington DC 2011, p. 4.

${ }^{24}$ Ibidem, p. 21

25 R.J. Heuer, Taxonomy of Structured Analytic Techniques, 2008, p. 4, http://www.pherson.org/ wp-content/uploads/2013/06/03.-Taxonomy-of-Structured-Analytic-Techniques_FINAL.pdf, accessed 11.10. 2016. 
analysis allows minimizing the negative effects of a traditional analysis process and overcoming cognitive limitations. Analysts not trained in statistics, higher mathematics or science may use the techniques of structured analysis. Training analysts to use these techniques can be conducted in the work environment - career development courses, etc. Recently, even some civilian universities have offered educational programmes on international security and analysis of information focusing on those techniques ${ }^{26}$.

Quantitative methods using expert-generated data are the third category. Analysts often do not have any, or a sufficient amount of, empirical data needed for an analysis. In the absence of this data a number of methods, especially those subjectively assessing the probability, are based on quantitative data generated by experts and their opinions. This category includes Bayesian inference, dynamic modelling and simulations. An analyst training in the use of these methods is based mainly on the education acquired at universities in the fields of mathematics, operations research, economics and marketing ${ }^{27}$.

The fourth and final category, quantitative methods using empirical data, includes econometric modelling as the main representative of this category. Empirical data is collected using a variety of technical means and sensors. Preparing analysts to use these methods is based on training in statistics, economics and science ${ }^{28}$.

None of these categories is to be considered better or worse. Each of them, depending on the circumstances, may be helpful. Using several methods from different categories when solving an analytical problem should be an accepted norm.

\section{Genesis, Essence and Concept of Structured Analysis and Analytic Techniques}

At the beginning of this century the analytical intelligence community in the United States, forced by spectacular intelligence failures (two attacks on the World Trade Centre, for example) to explore new methods of analysis and training analysts, turned their attention to structured analytic techniques. Structured analysis and the techniques were recognized as a means to overcome the limitations of reasoning and cognitive biases that constitute "the Achilles' heel" of each analyst.

The term Structured Analysis Techniques was first used in the US intelligence circles in 2005. The origins of the concept to structure the process of information

\footnotetext{
${ }^{26}$ R.J. Heuer, R.H. Pherson, Structured ..., op.cit., p. 22

27 R.J. Heuer, Taxonomy ..., op.cit., p. 3.

${ }^{28}$ Ibidem.
} 
analysis dates back to the 80 s, when lecturer and intelligence analyst Jack Davis began to teach and write about the so-called "alternative analysis", which he defined as processing, analysis and evaluation of alternative hypotheses, and advocated basing analyses on the cultural aspects of the analysed state or community and, finally, conducting analysis and forecasting from the perspective of the analysed state, communities and policy makers ${ }^{29}$.

In 2004, a group of academics from the Sherman Kent $\mathrm{School}^{30}$, analysts' school of the Central Intelligence Agency, faced the task of updating the training programme so that they would reflect the new requirements set for the intelligence community and to better prepare their analysts ${ }^{31}$. During their work, Randy Pherson and Roger Z. George came to the conclusion that the term "alternative analysis" was inappropriate, because a variety of analytical techniques were grouped under this name, and also because of the erroneous belief among intelligence analysts that alternative analysis techniques were just an occasional diversity to be used for less important analytical problems. The wife of Randy Pherson coined the name Structured Analysis Techniques. In June 2005, the name was officially approved for use in the new programme and training materials of the Central Intelligence Agency ${ }^{32}$.

Structured analytic techniques help to rigorously conduct analysis and the thought processes associated with it. Those techniques ensure that the main assumptions, cognitive biases and fallacies are not automatically accepted, but are critically examined and their impact on the result of the analysis is assessed.

The essence of the structured analysis method and structured analytical techniques is to organise massive amounts of data and information analysts have to tackle in the course of solving analytical problems, by breaking down these problems into components and then applying appropriate processes such as techniques to analyse the components of a given problem ${ }^{33}$.

Structured analysis is a process during which the internal thought processes occurring in an analyst's mind, while working on analytical problems, are expressed in the form of a structured, transparent and reproducible model. The resulting model allows analysts to share the results of an analysis as well as methods and details of the process itself. Furthermore, it allows other analysts to complement it and share the critique on both the results of analysis and the analytic process ${ }^{34}$.

${ }^{29}$ R.J. Heuer, R.H. Pherson, Structured ..., op.cit., p. 8.

${ }^{30}$ More on the Sherman Kent School at: https://www.cia.gov/offices-of-cia/intelligence-analysis/ training-resources.html, accessed 20.102016.

31 R.J. Heuer, R.H. Pherson, Structured ..., op. cit., p. 9.

32 Ibidem, p. 10.

${ }^{33}$ Ibidem, p. 4.

34 Ibidem. 
Correspondingly, structured analytic techniques constitute a unique set of tools which are used to assist analysts in mitigating, and offsetting the negative effects of their own mistakes and cognitive biases may be impairing the results of their analysis. The most important feature of structured analytical techniques is the ability to decompose the thought process of an analyst or team of analysts in a manner allowing its verification, documentation and criticism. Structured analytical techniques necessitate dialogue and communication between analysts enabling the use of the experience and knowledge of other analysts. In addition, the structured analysis process conducted in groups helps to avoid the "groupthink" that is characteristic of so-called "small groups" 35 .

\section{Structured Analytic Techniques and the Intelligence Cycle}

The Intelligence Cycle consists of a systematic sequence of activities, by which intelligence corresponding to the needs of customers is gathered (see Figure 4). NATO's doctrine AJP-2 defines the Intelligence Cycle as "the sequence of activities whereby information is obtained, assembled, converted into intelligence and made available for users" ${ }^{36}$. The Intelligence Cycle is designed to provide answers to the current questions and analytical problems mainly at the tactical level and is based on the customer-service provider relationship. The client asks for information on a specific topic and the service provider plans, collects, analyses and delivers them to the customer ${ }^{37}$. In recent years, the term 'intelligence process' has gained more popularity over the term the 'Intelligence Cycle', because it was recognized that it is not really a cycle but rather a process ${ }^{38}$.

AJP-2 defines analysis as a "third step in the processing phase where information is subjected to review in order to identify significant facts for subsequent interpretation"39.

The essence of the processing phase of the Intelligence Cycle is the transformation of the collected information into intelligence that meets the needs of customers. The remaining steps in the processing phase are:

${ }^{35}$ Ibidem, p. 23

${ }^{36}$ AJP-2, Allied ..., op.cit., p. 4-1.

${ }^{37}$ A. Roling, Strategic Intelligence for the $21^{\text {st }}$ Century. The Mosaic Method, CPI Group, Oxford, 2013, pp. 36-37.

${ }^{38}$ H. Prunckun, Scientific Methods of Inquiry for Intelligence Analysis, Second Edition, Rowman \& Littlefield Publishers, London, 2015, p. 7.

${ }^{39}$ AJP-2, Allied ..., op.cit., p. 4-6. 
Collation - this stage of the processing phase relies on organizing, systematizing and connecting information to facilitate further processing ${ }^{40}$.

Evaluation is the step in which both the information itself and its source are evaluated in terms of reliability and quality ${ }^{41}$.

Interpretation is the closing step in the processing phase and its goal is to clarify the importance of processed data in the light of the problem in question ${ }^{42}$ (see Figure 5).

\section{Figure 4. NATO's Intelligence Cycle}

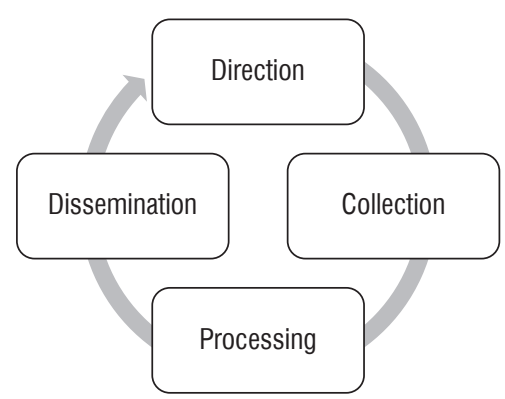

Source: AJP-2, Allied Joint Doctrine for Intelligence, Counter-Intelligence and Security, Edition A version 1, 2014, p. 4-1.

Figure 5. Processing phase of the Intelligence Cycle

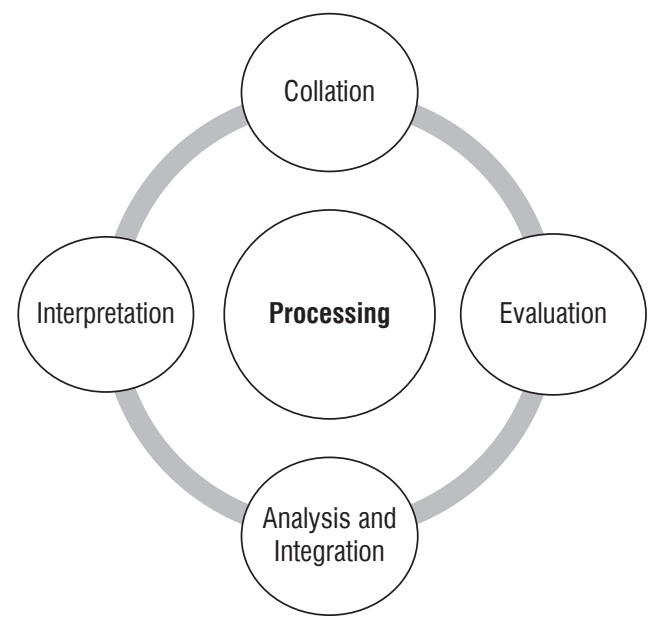

Source: AJP-2, Allied Joint Doctrine for Intelligence, Counter-Intelligence and Security, Edition A version 1, 2014, p. 4-4.
40 Ibidem, p. 4-4.
41 Ibidem. p. 4-5.
42 Ibidem, p. 4-6. 
Inspecting the definition of analysis and the processing phase provided by the authors of AJP -2 one can easily notice those two concepts are narrowed down in comparison to the analytic process defined by R.J. Heuer. Such a narrow understanding of analysis often confuses analysts when they are introduced to structured analysis and Heuer's analytic process. The intelligence analysis process restricted only to the processing phase excludes from its scope activities that are indigenous to that process. The analytic process is present in all the phases of the intelligence process and should be treated as a process within a process conducted simultaneously.

Comparing the steps of the processing phase to the essence of structured analysis and categories of structured analytic techniques, it should be noted that the structured techniques do not support analysis within the Intelligence Cycle in doctrinal terms, but rather support the entire information processing phase (see Figure 6).

Figure 6. Processing phase steps and structured techniques categories

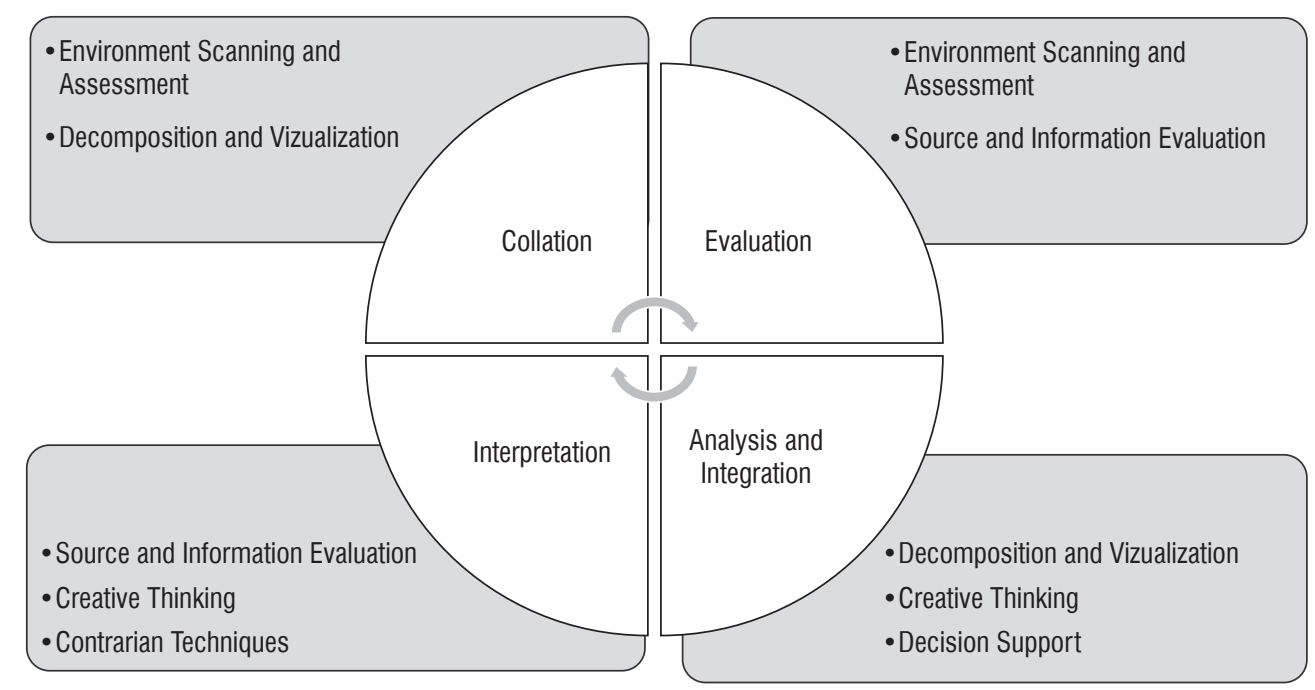

Source: the author's own study.

Structured analytic techniques often support other phases of the Intelligence Cycle, such as: orientation, in the case of scenario evaluation techniques (Backcasting) and hypothesis testing (Analysis of Competing Hypotheses), or when we use them to develop indicators or identify intelligence gaps and information requirements. Structured Analysis and its techniques also support processes and activities outside the Intelligence Cycle, such as decision-making process. 


\section{Structured Analytic Techniques Taxonomy}

Structured analytical techniques help to reduce the negative impact of cognitive biases, thought patterns and erroneous assumptions on the process of analysis. These techniques can support decision-making, and facilitate the exchange of information and cooperation between analysts and groups of analysts. The use of structured analytical techniques is intended to improve the process of analysis and increase its efficiency and effectiveness. Reviewing the literature on the topic, one can encounter different variants of the taxonomies of structured analytic techniques. Albeit hardly exhaustive, a review of the most popular taxonomies of structured techniques is presented as follows.

R.J. Heuer and R.H. Pherson systemize structured analytic techniques using the criterion of how the various techniques help overcome fallacies and cognitive biases in order to reach a solution to the analytical problem ${ }^{43}$. They classify those techniques into the following eight categories: decomposition and visualization techniques, idea generation techniques, scenarios and indicators techniques, hypothesis generation and testing techniques, assessment of cause and effect techniques, challenge analysis techniques, conflict management and decision support (see Figure 7).

Figure 7. R.J. Heuer's and R.H. Pherson's taxonomy

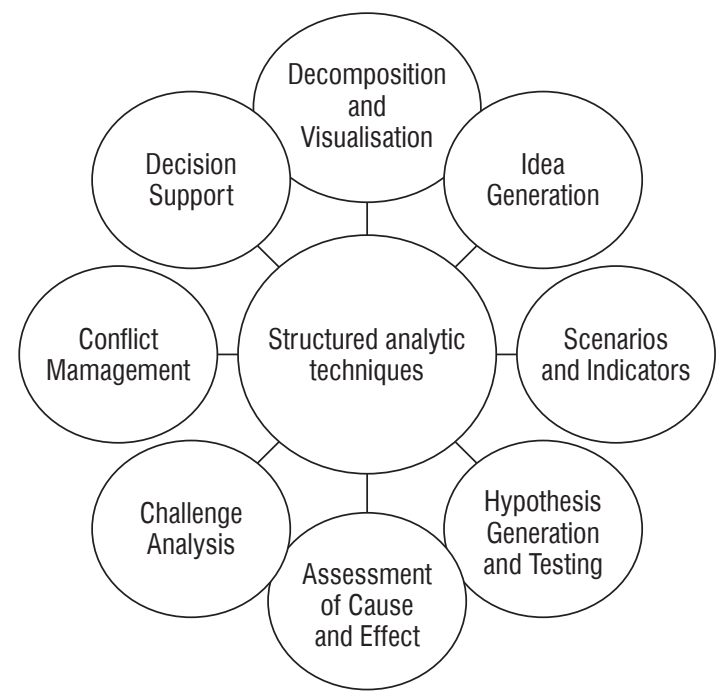

Source: the author's own study based on R.J. Heuer, R.H. Pherson, Structured Analytic Techniques for Intelligence Analysis, CQ Press, Washington DC 2011.

${ }^{43}$ R.J. Heuer, R.H. Pherson, Structured ..., op.cit., p. 24. 
A different taxonomy of structured analytic techniques is presented in the Central Intelligence Agency primer for analysts: A Tradecraft Primer: Structured Analytic Techniques for Improving Intelligence Analysis. In this publication, structured techniques are grouped according to the results achieved when a specific technique is used, and divided into diagnostic techniques, contrarian techniques and imaginative thinking techniques (see Figure 8).

Figure 8. Kent School taxonomy of structured analytic techniques

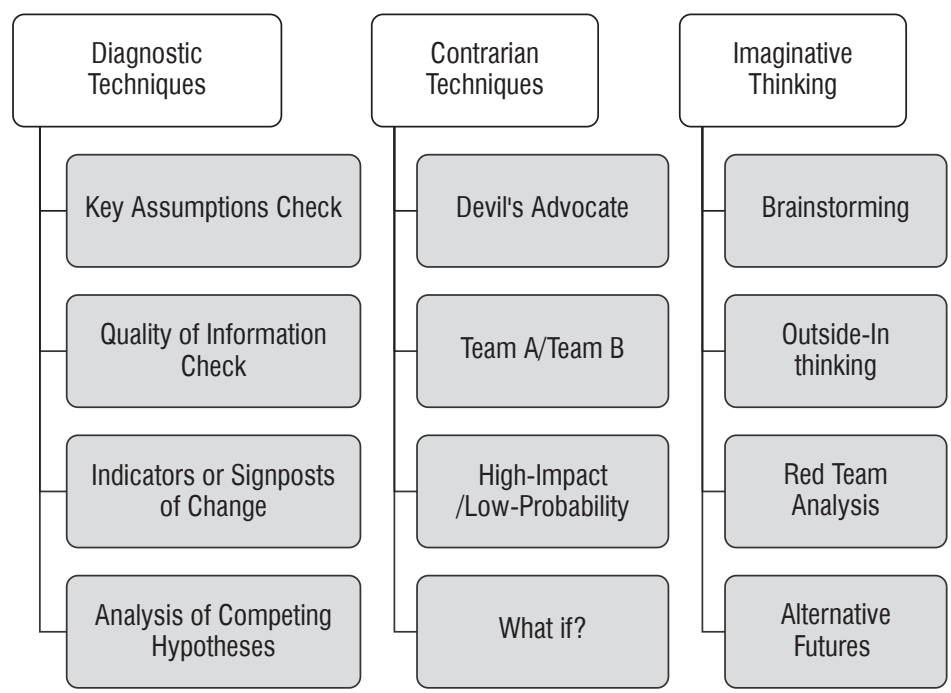

Source: the author's own study based on A Tradecraft Primer: Structured Analytic Techniques for Improving Intelligence Analysis, Sherman Kent School, CIA 2005.

Another taxonomy of structured analytic techniques the author has encountered classifies them into: focus techniques, contrarian techniques, quantitative techniques, and visual techniques (see Figure 9).

Considering the analytic spectrum ${ }^{44}$ structured analytic techniques can be divided into techniques that describe, techniques that explain, techniques that evaluate and techniques that estimate (see Fig. 10).

Monitoring, description and assessment of the environment in relation to which an analysis is or will be conducted, and an assessment of effects that the existing factors in this environment have, or may have, on an analytical problem, are a starting point, and a basis for solving that analytical problem. Without a thorough environmental assessment it is not possible to conduct an effective analysis. Moreover, the quality

${ }^{44}$ See Figure 2, Analytic spectrum. 
and accuracy of conclusions and hypotheses depend on the credibility of sources and the quality and usefulness of the information provided by them, as well as the subjective evaluation of sources and information by an analyst. Therefore, it is important that the analyst conducts his/her own assessment of sources and information, independent of the assessment provided by sensors and pre-processing operators. This independent analysis should help the analyst avoid cognitive biases, prejudices and hasty generalizations about the sources and information. The author advocates that the taxonomies of structured analytical techniques should be complemented by environment scanning and assessment techniques together with source and information evaluation techniques as separate categories. Those techniques are essential for completing the descriptive and explanatory stages of the analytic spectrum.

Figure 9. Focus, contrarian, quantitative and visual techniques

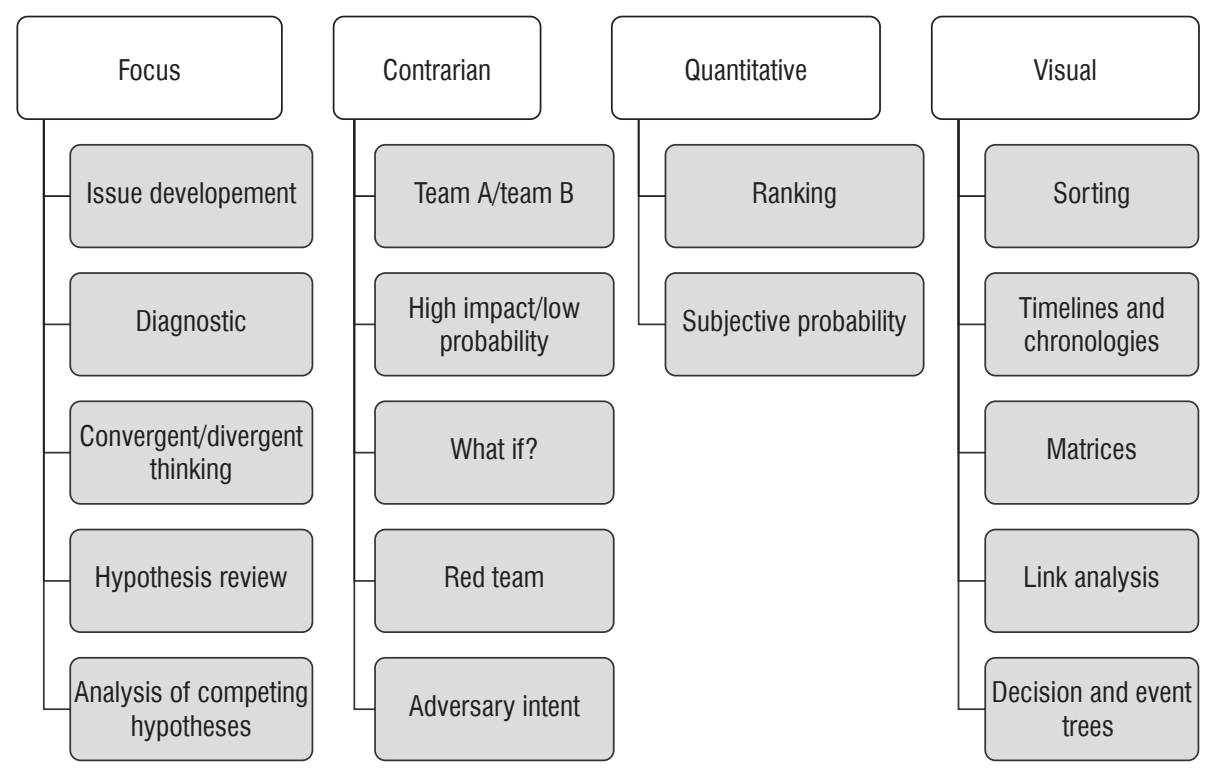

Source: the author's own study.

Considering the above observations, the author proposes the following taxonomy of structured analytic techniques ${ }^{45}$ (see Figure 11). The presented classification and selection of techniques resulted from the author's attempts to improve the teaching and learning process of the analysts who were novice to structured analytic

45 The following taxonomy and selection of techniques for each category is based on the review of the literature augmented by the author's professional experience. 
techniques and, from the author's experience, aids novice analysts in understanding the purpose of used techniques and selecting the best-suited technique for a given analytic problem. Experienced anlysts would, undoubtedly, modify or even develop their own classifications and selection of prefered techniques.

Figure 10. Structured analytic techniques taxonomy according to K. Hibbs Phearson and R.H. Pherson

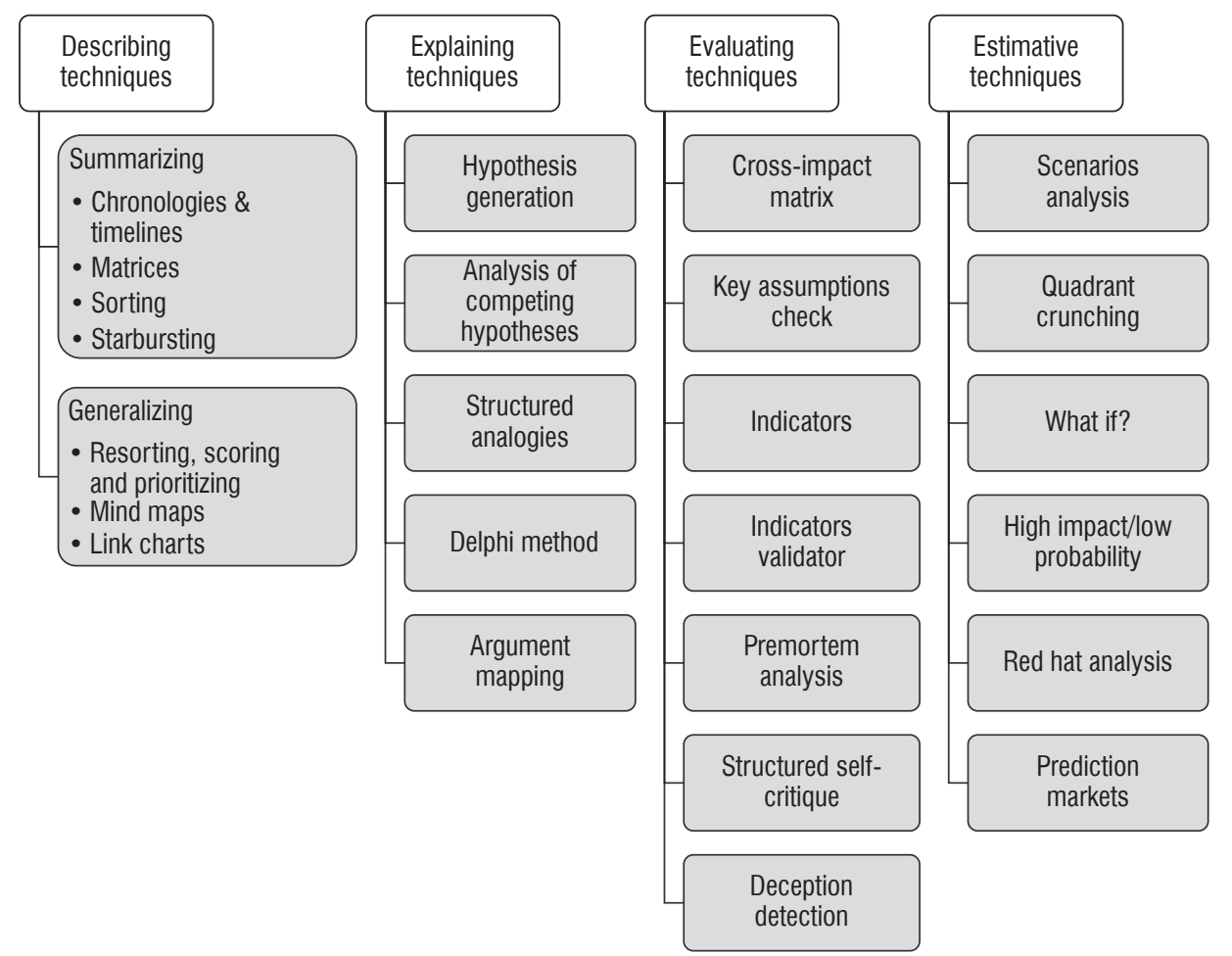

Source: K. Hibbs Pherson, R.H. Pherson, Critical Thinking for Strategic Intelligence, SAGE/CQPress, Los Angeles 2013.

Environment scanning and assessment techniques are used to describe the environment, assess and analyse it in relation to which the analyses are or will be conducted, and to assess the effects that this environment may have on a particular analytical problem. These techniques include the following: PMESII (Political, Military, Economic, Social, Infrastructure and Information), STEMPLES (Social, Technological, Environmental, Military, Political, Legal, Economic, Security), and SWOT (Strengths, Weaknesses, Opportunities, Threats). These techniques can also be used in the Intelligence Preparation of the Battlefield process to define the battlefield and describe the battlefield's effects. 
Source and information evaluation techniques are used to evaluate and supplement the knowledge of the intelligence information and its sources. Using those techniques, an analyst can avoid cognitive biases, prejudices and hasty generalizations about information and its sources. This category includes techniques: source check, quality-of-information and relevance check.

\section{Figure 11. Structured analytic techniques categories}

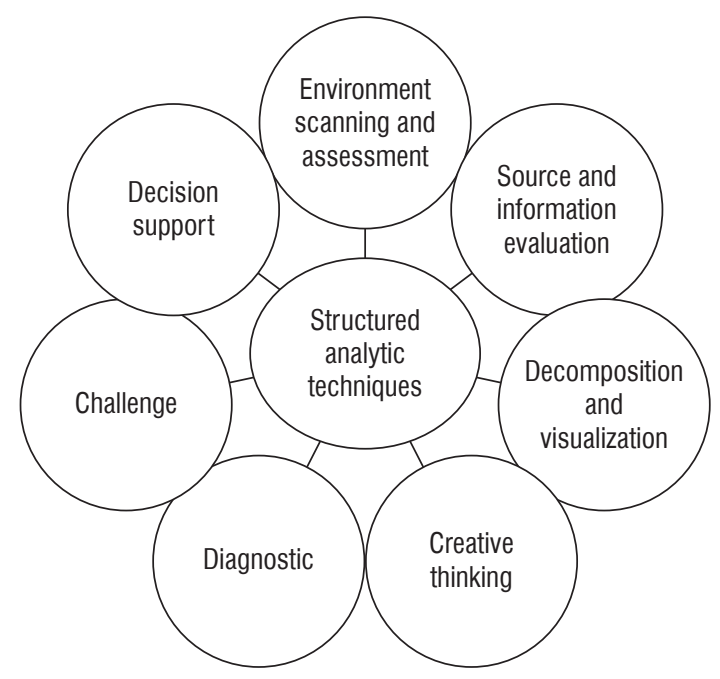

Source: the author's own study.

Decomposition and visualization techniques facilitate overcoming the limitations of the human mind in terms of the amount of information possible to be processed. The restricted number of bits of information that the human brain can process at a given time makes it virtually impossible not to omit particular facts and information in the analysis process. Decomposition techniques include: customer checklist, AIMS (Audience, Issue, Message, and Storyline), and issue redefinition. On the other hand, visualization techniques are comprised of: Sorting, Weighted Ranking, Filtering, Chronologies and Timelines, Network Analysis, Event and Scenario Trees, and Venn Analysis.

Creative thinking techniques are used to develop alternative outcomes, perspectives and points of view ${ }^{46}$. These techniques can be categorized by:

${ }^{46}$ A Tradecraft Primer: Structured Analytic Techniques for Improving Intelligence Analysis, Sherman Kent School, CIA 2005, p. 5. 
1. Idea generation techniques, which consist of: Structured Brainstorming, Nominal Group Technique, Starbursting, Quadrant Crunching, and Convergent and Divergent Thinking.

2. Scenarios generation techniques, which include: Alternative Futures, Simple Scenarios, Multiple Scenarios Generation, Cone of Plausibility, and Simplified Red Team.

3. Hypothesis generation techniques, which include: Simple Hypotheses, Multiple Hypotheses Generation, and Quadrant Hypothesis Generation.

4. Indicators is the last creative thinking category, and consists of Indicators Technique. Diagnostic techniques are used to verify the hypotheses, scenarios and assumptions developed using creative thinking techniques. They are also used to identify, formulate and define analytical arguments, assumptions and information gaps ${ }^{47}$. Diagnostic techniques are grouped into three categories:

1. Indicators validation consists of a single technique - Indicators Validator.

2. Hypothesis testing includes the techniques of: Diagnostic Reasoning, Analysis of Competing Hypotheses, Key Assumptions Check, Argument Mapping, and Hypotheses Mapping.

3. Scenarios evaluation techniques comprise of: Backcasting, Alternative Futures, Multiple Scenarios Generation, Simple Scenarios, and Simplified Red Team.

The essence of contrarian techniques is to question the current line of thinking ${ }^{48}$, and previous hypotheses in order to generate alternative viewpoints, hypotheses and scenarios. Contrarian techniques include: Structured Self-Critique, Premortem Analysis, What If?, High Impact/ Low Probability and the Devil's Advocacy.

Decision support techniques are used to support and facilitate planning and decision-making processes. This category includes the following techniques: ProsCons-Fixes, Decision Matrix, Force Fields Analysis, SWOT and Decision Trees.

\section{Conclusion}

Analysis is certainly one of the most difficult and yet critical function in any organization. An analyst follows processes, events, people, etc., and based on this formulates hypotheses explaining the observed phenomena. Then, using those hypotheses (s)he is trying to predict future events. In order to perform those functions, an

47 Ibidem.

48 Ibidem. 
effective analysis requires experience, reliable information, intuition and models, techniques and methodologies ${ }^{49}$.

In analysts' environment, one can meet with arguments that structured techniques are not useful or effective, especially when compared with automated systems of data and information analysis, or totally unsuitable for the analysis of "Big Data". It should be noted, however, that regardless of the available systems and technical tools, and regardless of the type of analysed information or data, analytic techniques, and especially structured analytic techniques, should constitute basic skills acquired during analyst initial training.

As mentioned previously, a lot of effort is spent gathering information, while underestimating and ignoring the analysis itself. It can be seen clearly when comparing available commercial tools and services for information collection with the offered services and tools for the analysis and processing of information. B.E. Bensoussan and C.S. Fleisher list the following reasons why analytic techniques are underestimated and even avoided by decision makers:

1. "Analysis is difficult for most people.

2. Hardly anybody analyses their analysis.

3. Few people have publicly recognized or established analysis expertise.

4. Few frameworks exist for understanding how the analysis component can be managed as an integral part of the larger decision making process" 50 .

In the author's opinion, structured analytic techniques, being already in use in defence, law enforcement and intelligence communities, aid in overcoming the aforementioned problems, help analysts improve analyses of competition, market and environment, and facilitate strategy development and decision-making. The use of structured techniques improves the analysis process and makes it easier to control. In addition, these techniques help to reduce the impact of heuristics and cognitive biases on the outcome of analysis, while keeping in mind that no technique can replace the experience and knowledge of an analyst.

Taxonomy and selection techniques presented in this article are a proposal based on the author's experience, and the review of the subject's literature. The goal of the presented taxonomy is to facilitate an analyst's training and the selection of the proper technique to solve a given analytical problem.

\footnotetext{
${ }^{49}$ B.E. Bensoussan, C.S. Fleisher, Analysis..., op.cit., p.15.

${ }^{50}$ Ibidem, pp. 13-14.
} 


\section{Bibliography}

1. AJP-2, Allied Joint Doctrine for Intelligence, Counter-Intelligence and Security, Edition A version 1, 2014.

2. A Tradecraft Primer: Structured Analytic Techniques for Improving Intelligence Analysis, Sherman Kent School, CIA 2005.

3. Bensoussan B.E., Fleisher C.S., Analysis Without Paralysis. 12 Tools to Make Better Strategic Decisions, FT Press Upper Saddle River, New Jersey 2013.

4. Fischhoff B, Chauvin C., Intelligence Analysis: Behavioral and Social Scientific Foundations. The National Academies Press, Washington DC 2011.

5. Forget information overload...the real challenge is content intelligence. Research Summary, MindMeetre, February 2014; http://www.mindmetreresearch.com/wp-content/ uploads/2014/05/Paper_1_2014_FINAL1.pdf, accessed 20.10.2016.

6. Heuer R.J., Psychology of Intelligence Analysis, Center for the Study of Intelligence, CIA, 1999.

7. Heuer R.J., Taxonomy of Structured Analytic Techniques, http://www.pherson.org/wpcontent/uploads/2013/06/03.-Taxonomy-of-Structured-Analytic-Techniques_FINAL. pdf, accessed 11.10. 2016.

8. Heuer R.J., Pherson R.H., Structured Analytic Techniques for Intelligence Analysis, CQ Press, Washington DC 2011.

9. Hibbs Pherson K., Pherson R.H., Critical Thinking for Strategic Intelligence, SAGE/ CQPress, Los Angeles 2013.

10. Intelligence Analysis, RAND Corporation, http://www.rand.org/topics/intelligenceanalysis.html, accessed 26.10.2016.

11. Mangio C.A., Intelligence Analysis: Once Again, Air Force Research Laboratory Interim Report, February 2008, http://www.dtic.mil/docs/citations/ADA520278, accessed 10.01.2017.

12. Miller G.A., The Magical Number Seven, Plus or Minus Two, http://web.archive.org/ web/20080728075223/www.musanim.com/miller1956/, accessed 24.02.2015.

13. Palladino L.J., Find Your Focus Zone: An Effective New Plan to Defeat Distraction and Overload, Atria Books, New York 2007.

14. Prunckun H., Scientific Methods of Inquiry for Intelligence Analysis, $2^{\text {nd }}$ edition, Rowman \& Littlefield Publishers, London, 2015.

15. Roling A., Strategic Intelligence for the $21^{\text {st }}$ Century. The Mosaic Method, Oxford CPI Group, Oxford 2013.

16. Słownik języka polskiego PWN. http://sjp.pwn.pl, accessed 21.10. 2016.

17. Toffler A., Future Shock, Random House, New York 1970. 